\title{
Population substructure in Cache County, Utah: the Cache County study
}

\author{
Aaron R Sharp ${ }^{1}$, Perry G Ridge1, Matthew H Bailey ${ }^{1}$, Kevin L Boehme', Maria C Norton²,3,6, JoAnn T Tschanz ${ }^{3,6}$, \\ Ronald G Munger ${ }^{4,6}$, Christopher D Corcoran ${ }^{5,6}$, John SK Kauwe ${ }^{1 *}$, \\ Alzheimer's Disease Neuroimaging Initiative $(\mathrm{ADNI})^{7}$
}

From The 10th Annual Biotechnology and Bioinformatics Symposium (BIOT 2013)

Provo, UT, USA. 5-6 December 2013

\begin{abstract}
Background: Population stratification is a key concern for genetic association analyses. In addition, extreme homogeneity of ethnic origins of a population can make it difficult to interpret how genetic associations in that population may translate into other populations. Here we have evaluated the genetic substructure of samples from the Cache County study relative to the HapMap Reference populations and data from the Alzheimer's Disease Neuroimaging Initiative (ADNI).

Results: Our findings show that the Cache County study is similar in ethnic diversity to the self-reported "Whites" in the ADNI sample and less homogenous than the HapMap CEU population.

Conclusions: We conclude that the Cache County study is genetically representative of the general European American population in the USA and is an appropriate population for conducting broadly applicable genetic studies.
\end{abstract}

\section{Background}

Cryptic population differences due to heterogeneity have confounding effects on association studies, especially when analyzing complex traits and gene-gene interactions. It is also possible that genetic associations that are observed in very homogeneous populations may not be generalizable to other populations.

The Cache County study is a large longitudinal cohort study of memory, health, and aging that was initiated in 1994. This sample of 5,092 individuals represents approximately $90 \%$ of the Cache County population aged 65 and older at that time. These data are a valuable resource in genetic studies of Alzheimer's disease (AD), as well as otherdiscussion forms of dementia [1]. The founding populations and migrations to Utah by early members of the Church of Jesus Christ of Latter Day Saints have been studied extensively[2-4]. These studies have concluded that due to the large founding population, high rates of

'Department of Biology, Brigham Young University, Provo, Utah, USA Full list of author information is available at the end of the article gene flow and diversity of source populations the Utah population has allele frequencies that are quite similar to the general European American population in the United States. As it was collected from one county in Utah, there have been questions as to how the genetic diversity in the Cache County sample compares to that of more broadly collected European American samples and whether that diversity affects the validity or generalizability of results obtained from the Cache County study.

The Cache County study is a large longitudinal cohort study of memory, health, and aging that was initiated in 1994. This sample of 5,092 individuals represents approximately $90 \%$ of the Cache County population aged 65 and older at that time. These data are a valuable resource in genetic studies of Alzheimer's disease (AD), as well as other forms of dementia [1]. The founding populations and migrations to Utah by early members of the Church of Jesus Christ of Latter Day Saints have been studied extensively[2-4]. These studies have concluded that due to the large founding population, high rates of gene flow and diversity of source populations the Utah population has 
allele frequencies that are quite similar to the general European American population in the United States. As it was collected from one county in Utah, there have been questions as to how the genetic diversity in the Cache County sample compares to that of more broadly collected European American samples and whether that diversity affects the validity or generalizability of results obtained from the Cache County study.

The purpose of this study is to compare the genetic structure of the Cache County study, which was collected from one county in northern Utah, to that of the Alzheimer's Disease Neuroimaging Initiative, a sample that was collected from several sites around the USA, and data from several other populations from the International HapMap Project.

\section{Methods}

\section{Sample collection and genotyping}

The Cache County study originally included 5,092 permanent residents of Cache County over the age of 64 . Details of collection methods and demographics of the Cache County samples have been reported previously [1]. Briefly, samples underwent four triennial waves of data collection in a multi-stage dementia screening and assessment protocol. DNA was obtained from blood and buccal swabs as described by Breitner et al. [1] All study procedures were approved by the Institutional Review Boards of Utah State, Duke and Johns Hopkins universitys. DNA from 506 Cache County study participants was genotyped using the Illumina OmniExpress chip; DNA from 234 others was genotyped using the Illumina $2.5 \mathrm{M}$ chip.

The broader population data with which we will compare the Cache data was collected by The International HapMap Project [5] and the Alzheimer's Disease Neuroimaging Initiative (ADNI) [6]. The International Hapmap Project (phase 3, draft release 2) consists of SNP genotyping data from the populations in Table 1 [5]. The data were collected using two genotyping platforms: Illumina
Human $1 \mathrm{M}$ by the Wellcome Trust Sanger Institute and Affymetrix SNP 6.0 by the Broad Institute. They are available for download from http://hapmap.ncbi.nlm.nih.gov/ downloads/genotypes/2009-01_phaseIII/plink_format.

The ADNI samples are part of a longitudinal study designed to measure the progression of mild cognitive impairment (MCI) and early AD [6]. The primary goal of ADNI has been to test whether serial magnetic resonance imaging (MRI), PET, other biological markers, and clinical and neuropsychological assessment can be combined to measure the progression of MCI and early AD (PI: Michael W. Weiner, M.D., VA Medical Center and University of California - San Francisco). ADNI is the result of efforts of many co- investigators from a broad range of academic institutions and private corporations, and subjects have been recruited from over 50 sites across the U. S. and Canada. For up-to-date information, see http:// www.adni-info.org. Data for the present analysis were downloaded from the ADNI web site in March 2013. We used self-reported race to label three ethnic groups within the ADNI samples, Black/African-American (ADNI1), White (ADNI2), or Asian (ADNI3).

\section{Data preparation and quality control}

For the distance-based clustering, the following data preparation steps were taken. First, all palindromic markers (alleles $\mathrm{A} / \mathrm{T}$ or $\mathrm{G} / \mathrm{C}$ ) were excluded to correct for strand differences between datasets (120,541 markers excluded). We then excluded all markers that were not genotyped in at least two of the three data sets $(342,603$ markers included). Finally, we removed all markers with genotyping rates lower than 95\% across all individuals (263,883 markers included). Individuals with genotyping rates of lower than $95 \%$ for the included markers were excluded from the analysis. The final set consisted of 2,274 individuals, 400 from Cache, 626 from ADNI, and 1,248 from the various HapMap populations.

For the model-based clustering, we compared Cache to only two populations (CEU and ADNI2), because

Table 1 HapMap populations with Abbreviations

\begin{tabular}{ll}
\hline Population & Abbreviation \\
\hline African ancestry in Southwest USA & ASW \\
CEPH (Utah residents with ancestry from northern and western Europe) & CEU \\
Han Chinese in Beijing, China & CHB \\
Chinese in Metropolitan Denver, Colorado & CHD \\
Gujarati Indians in Houston, Texas & GIH \\
Japanese in Tokyo, Japan & JPT \\
Luhya in Webuye, Kenya & LWK \\
Mexican ancestry in Los Angeles, California & MEX \\
Maasai in Kinyawa, Kenya & MKK \\
Tuscan in Italy & TSI \\
Yoruban in Ibadan, Nigeria & YRI \\
\hline
\end{tabular}


they appeared most similar in the distance-based method. This included a total of 1,155 samples. Pruning of markers to minimize redundant information due to linkage disequilibrium was done using the following parameters in PLINK: $\mathrm{r}^{2}>.10$ in 50 SNP windows, incremented by 5 SNPs across the genome (61616 markers included).

\section{Methods for distance and model-based clustering analysis} The distance-based method of comparison is a standard classical (metric) multi-dimensional scalar analysis of pairwise identity by state distances. Distances were calculated and scaling was performed using the PLINK whole genome association analysis toolset [7].

In addition to distance scaling, Bayesian modeling techniques were leveraged to identify clustering of samples from Cache and the other European-American populations. The model-based clustering method was provided using the program Structure [8]. Burn-in duration was set at 5,000 repetitions with a run duration of 10,000 repetitions per software recommendations[8]. We used an admixture type ancestry model due to the generally admixed nature of the European American population and specified for a model of correlated allele frequency across result populations. The statistic of interest generated by Structure is a probability, $\operatorname{Pr}(\mathrm{Z} \mid \mathrm{P}, \mathrm{X})$, where $\mathrm{Z}$ is a vector containing the assignment to a cluster of each individual, $\mathrm{P}$ specifies the frequency of each allele at each locus (a statistic that is calculated by Structure to characterize each cluster), and $x$ is the given genotypes of the sampled individuals.

\section{Results}

Results from the distance-based clustering approach Results indicate that samples from the Cache County study cluster very closely with other European American samples (ADNI2 and CEU; Figure 1). In addition, the Cache County samples exhibit a range of diversity that is similar to that of self reported whites from the ADNI sample (ADNI2; Figure 2).

\section{Results from the model-based clustering approach}

Structure results indicate that the observed range of genotypes is most likely to have occurred if the individuals came from two separate populations, as opposed to any other number of populations from one to five (table 2). Additionally, the majority of individuals came from only one of the two proposed clusters (table 3).

\section{Discussion}

The Cache County study has provided important information about genetic factors that influence aging and Alzheimer's disease [1,9-13]. The results reported here indicate that despite being collected from a single county in northern Utah, these samples are comparable in levels of diversity to those of self-reported European-Americans collected at multiple sites across the United States. In addition, we observed no evidence of problematic population stratification between the Alzheimer's disease cases and non-demented controls in the Cache samples. This indicates that association studies in this population are unlikely to produce type-I error due to population stratification.

The distance-based clustering method identified similarity between the majority of European-American individuals, including those from Cache County, CEU, and ADNI2. The model-base clustering method found the optimum solution to be two clusters, one small cluster with a small number of outliers from each of the three samples (Cache, ADNI2, and CEU) and a larger cluster that included the vast majority of samples.

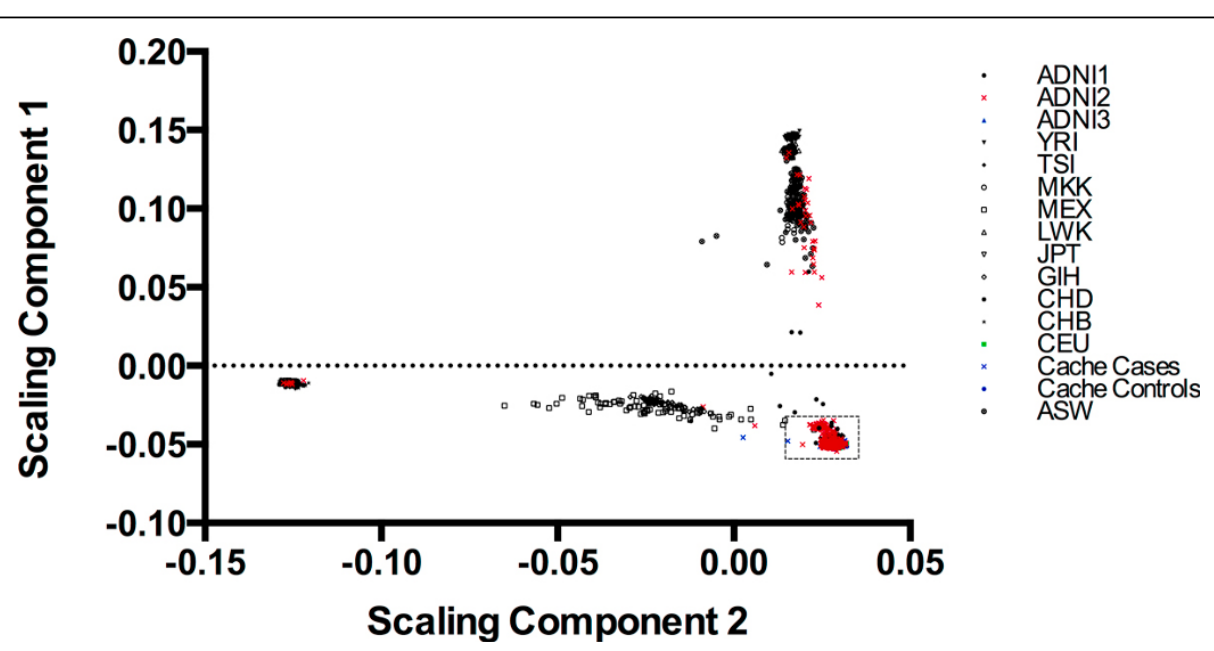

Figure 1 All individuals with scaling components. ADNI1 refers to individuals participating in the ADNI study who self-identified as "Black/ African-American", ADNI2 refers to those who self-identified as "White", and ADNI 3 refers to those who self-identified as "Asian". All other abbreviations are from Table 1. In Cache samples, case or control status refers to clinical diagnosis of Alzheimer's disease. Dashed box designates the region displayed in figure 2 and includes the ADNI2, CEU, Cache Cases and Controls. 


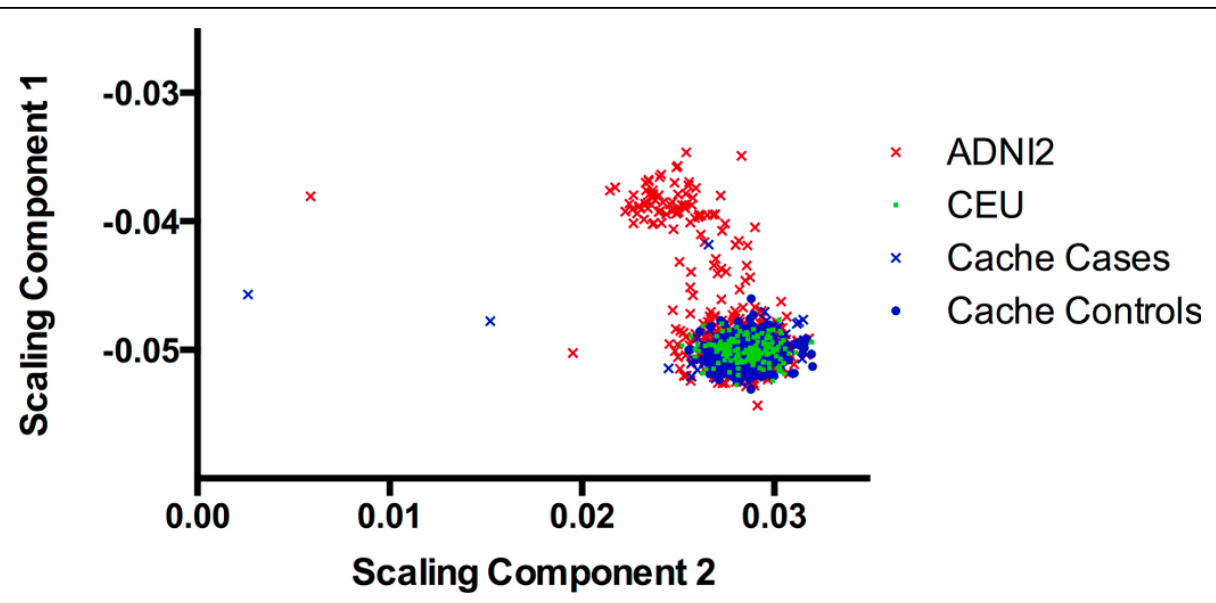

Figure 2 Detail from Figure 1. ADNI2 refers to those in ADNI who self reported their ethnicity as "White". CEU are the CEPH families from the HapMap Project and Cache cases and controls are with respect to Alzheimer's disease diagnosis.

Table 2 Log of probability of (k) from the combined ADNI2, CEU and Cache County Samples calculated using Structure.

\begin{tabular}{ll}
\hline Prior population estimate $(\mathbf{k})$ & $\ln (\mathbf{P}(\mathbf{Z} \mid \mathbf{P}, \mathbf{X}))$ \\
\hline 1 & $-73,240,718.0$ \\
2 & $-73,000,731.9$ \\
3 & $-74,033,402.2$ \\
4 & $-87,968,158.6$ \\
5 & $-136,266,205.0$ \\
\hline
\end{tabular}

Table 3 Cluster assignments from structure.

\begin{tabular}{lll}
\hline $\begin{array}{l}\text { Sample } \\
\text { population }\end{array}$ & $\begin{array}{l}\text { Proportion of individuals } \\
\text { assigned to minor result } \\
\text { cluster }\end{array}$ & $\begin{array}{l}\text { Proportion of individuals } \\
\text { assigned to major result } \\
\text { cluster }\end{array}$ \\
\hline Cache & .001 & .999 \\
ADNI2 & .060 & .940 \\
CEU & .001 & .999 \\
\hline
\end{tabular}

These data suggest that the population substructure in the Cache County study is comparable to that of other European-American samples that are more broadly collected, (i.e. the ADNI samples). In addition, these findings are consistent with the results reported previously on the larger population of the early population of the state of Utah [2-4]. We conclude that despite concerns about the limited geographic range of sample collection in the Cache County study, the results of genetic studies in this population, such as gene discovery, validation, and estimates of population level effects, are as broadly applicable to other populations of European American ancestry as that of the Alzheimer's Disease Neuroimaging Initiative.

\section{Competing interests}

The authors declare no conflicts of interest or competing interests with regard to this work.

\section{Acknowledgements}

The authors gratefully acknowledge the individuals who participated in this study without whom this work would not have been possible. We also acknowledge Alzheimer's Disease Neuroimaging Initiative (ADNI) for making their data publically available for this and other work(www.loni.ucla.edu/ADNI), as such, the investigators within the ADNI contributed to the design and implementation of ADNI and/or provided data but did not participate in analysis or writing of this report. A complete listing of ADNI investigators can be found at: http://loni.ucla.edu//ADNI//Collaboration//ADNI_Authorship_list. pdf. Data collection and sharing for this project was funded by the ADNI (National Institutes of Health Grant U01 AG024904). ADNI is funded by the National Institute on Aging, the National Institute of Biomedical Imaging and Bioengineering, and through generous contributions from the following: Abbott; Alzheimer's Association; Alzheimer's Drug Discovery Foundation; Amorfix Life Sciences Ltd.; AstraZeneca; Bayer HealthCare; BioClinica, Inc;; Biogen Idec Inc.; Bristol-Myers Squibb Company; Eisai Inc.; Elan Pharmaceuticals Inc; Eli Lilly and Company; F. Hoffmann-La Roche Ltd and its affiliated company Genentech, Inc:; GE Healthcare; Innogenetics, N.V.; Janssen Alzheimer Immunotherapy Research \& Development, LLC.; Johnson \& Johnson Pharmaceutical Research \& Development LLC.; Medpace, Inc.; Merck \& Co., Inc.; Meso Scale Diagnostics, LLC.; Novartis Pharmaceuticals Corporation; Pfizer Inc; Servier; Synarc Inc.; and Takeda Pharmaceutical Company. The Canadian Institutes of Health Research is providing funds to support ADNI clinical sites in Canada. Private sector contributions are facilitated by the Foundation for the National Institutes of Health (http://www.fnih.org). The grantee organization is the Northern California Institute for Research and Education, and the study is coordinated by the Alzheimer's Disease Cooperative Study at the University of California, San Diego. ADNI data are disseminated by the Laboratory for Neuro Imaging at the Rev August 162011 University of California, Los Angeles. This research was also supported by NIH grants P30 AG010129, K01 AG030514, R01 HG02213, U01 AG024904, U01 HG006500, K24 AG027841, R01 HG005092, P50AG005146, and the Dana Foundation.

\section{Declarations}

This work was supported by the Alzheimer's Association (MNIRG-11-205368), the BYU Gerontology Program and the National Institutes of Health (R01-AG11380, R01- AG021136, P30-NS069329-01, R01-AG042611). The Institutional Review Boards of each appropriate institution approved sample and data collection. This article has been published as part of BMC Bioinformatics Volume 15 Supplement 7, 2014: Selected articles from the 10th Annual Biotechnology and Bioinformatics Symposium (BIOT 2013). The full contents of the supplement are available online at http://www.biomedcentral.com/ bmcbioinformatics/supplements/15/S7.

\section{Authors' details}

'Department of Biology, Brigham Young University, Provo, Utah, USA. ${ }^{2}$ Department of Family Consumer and Human Development, Utah State 
University, Logan, Utah, USA. ${ }^{3}$ Department of Psychology, Utah State University, Logan, Utah, USA. ${ }^{4}$ Department of Nutrition, Dietetics, and Food Sciences, Utah State University, Logan, Utah, USA. ${ }^{5}$ Department of Mathematics and Statistics, Utah State University, Logan, Utah, USA. ${ }^{6}$ Center for Epidemiologic Studies, Utah State University, Logan, Utah, USA. 'UCLA,

LA, USA.

Published: 28 May 2014

\section{References}

1. Breitner JC, Wyse BW, Anthony JC, Welsh-Bohmer KA, Steffens DC, Norton MC, Tschanz JT, Plassman BL, Meyer MR, Skoog I, et al: APOEepsilon4 count predicts age when prevalence of $A D$ increases, then declines: the Cache County Study. Neurology 1999, 53:(2):321-331.

2. O'Brien E, Rogers AR, Beesley J, Jorde LB: Genetic structure of the Utah Mormons: comparison of results based on RFLPs, blood groups, migration matrices, isonymy, and pedigrees. Hum Biol 1994, 66:(5):743-759.

3. McLellan T, Jorde LB, Skolnick MH: Genetic distances between the Utah Mormons and related populations. Am J Hum Genet 1984, 36:(4):836-857.

4. Jorde LB: The genetic structure of the Utah Mormons: migration analysis. Hum Biol 1982, 54:(3):583-597.

5. International HapMap C: The International HapMap Project. Nature 2003, 426:(6968):789-796.

6. Petersen RC, Aisen PS, Beckett LA, Donohue MC, Gamst AC, Harvey DJ, Jack CR, Jagust WJ, Shaw LM, Toga AW, et al: Alzheimer's Disease Neuroimaging Initiative (ADNI): clinical characterization. Neurology 2010, 74:(3):201-209.

7. Purcell S, Neale B, Todd-Brown K, Thomas L, Ferreira MA, Bender D, Maller J, Sklar P, de Bakker PI, Daly MJ, et al: PLINK: a tool set for whole-genome association and population-based linkage analyses. Am J Hum Genet 2007, 81:(3):559-575.

8. Falush D, Stephens M, Pritchard JK: Inference of population structure using multilocus genotype data: linked loci and correlated allele frequencies. Genetics 2003, 164:(4):1567-1587.

9. Guerreiro R, Wojtas A, Bras J, Carrasquillo M, Rogaeva E, Majounie E, Cruchaga C, Sassi C, Kauwe JS, Younkin S, et al: TREM2 variants in Alzheimer's disease. The New England journal of medicine 2013, 368: (2):117-127.

10. Peterson D, Munger C, Crowley J, Corcoran C, Cruchaga C, Goate AM, Norton MC, Green RC, Munger RG, Breitner JC, et al: Variants in PPP3R1 and MAPT are associated with more rapid functional decline in Alzheimer's disease: The Cache County Dementia Progression Study. Alzheimers Dement 2013.

11. Ridge PG, Maxwell TJ, Corcoran CD, Norton MC, Tschanz JT, O'Brien E, Kerber RA, Cawthon RM, Munger RG, Kauwe JS: Mitochondrial Genomic Analysis of Late Onset Alzheimer's Disease Reveals Protective Haplogroups H6A1A/H6A1B: The Cache County Study on Memory in Aging. PLoS ONE 2012, 7:(9):e45134.

12. Gonzalez J, Schmutz C, Munger C, Perkes A, Gustin A, Peterson M, MTW E, Norton MC, Tschanz JT, Munger RG, et al: Assessment of TREM2 R47H association with Alzheimer's disease in a population-based sample: The Cache County Study. Neurobiology of Aging 2013.

13. Ebbert MT, Ridge PG, Wilson AR, Sharp AR, Bailey M, Norton MC, Tschanz JT, Munger RG, Corcoran CD, Kauwe JS: Population-based Analysis of Alzheimer's Disease Risk Alleles Implicates Genetic Interactions. Biological psychiatry 2013.

doi:10.1186/1471-2105-15-S7-S8

Cite this article as: Sharp et al: Population substructure in Cache County, Utah: the Cache County study. BMC Bioinformatics 2014 15(Suppl 7):S8.

\section{Submit your next manuscript to BioMed Central and take full advantage of:}

- Convenient online submission

- Thorough peer review

- No space constraints or color figure charges

- Immediate publication on acceptance

- Inclusion in PubMed, CAS, Scopus and Google Scholar

- Research which is freely available for redistribution

Submit your manuscript at www.biomedcentral.com/submit
Biomed Central 